\section{Fernerkundung in der Hydrologie und Wasserwirtschaft}

Fernerkundungsmethoden haben in den letzten Jahren die Beschaffung hydrologisch-wasserwirtschaftlicher Grundlagen revolutioniert. Das ist wahrscheinlich am deutlichsten beim Airborne Laserscanning zu erkennen. Während noch vor wenigen Jahren Geländeaufnahmen aus Stereofotos bzw. terrestrisch erfolgten, wird heute Laserscanning im breiten Maßstab in ganz Österreich eingesetzt, um hochgenaue Geländemodelle zu berechnen. Eine unerreichte Datendichte von mehreren Punkten pro Quadratmeter sowie Höhengenauigkeiten von besser als $15 \mathrm{~cm}$ sind heute durchaus verwirklichbar. Als präzise geometrische Datengrundlage vor allem für hydrodynamische Simulationen von Überflutungsflächen sind topografische Daten aus Laserscanning heute nicht mehr wegzudenken. Zweifelsfrei hat hier die Industrie mit der Entwicklung moderner Laserscanner-Technologien einen wesentlichen Schritt gemacht. Doch die effiziente Verarbeitung und zutreffende Interpretation solcher Daten sowie der richtige Einsatz bei wasserwirtschaftlich-hydrologischen Fragestellungen ist äußerst schwierig, aber dennoch essentiell, um aussagekräftige Ergebnisse zu erhalten. Hier ist sowohl die Fernerkundung als auch die Hydrologie wissenschaftlich gefordert. Die überaus vielfältigen Forschungsaktivitäten auf diesem Gebiet in Österreich haben entscheidend dazu beigetragen, die Methoden praxisreif zu machen, allen voran das Christian-Doppler-Labor „Räumliche Daten aus Laserscanning und Fernerkundung“, etwa bei der Entwicklung von modernen Filtermethoden zur Fehlerkorrektur sowie Full-Waveform-Technologien. Auch auf hydrologischer Seite gibt es neue Algorithmen bei der Lösung der hydrodynamischen Gleichungen, für effiziente Netzgeometrien sowie für die Bestimmung von Modellparametern (z. B. Rauigkeit).

Im Vergleich zu der raschen Verbreitung von Airborne Laserscanning in Österreich sind andere Fernerkundungsmethoden, die direkt auf die Bestimmung hydrologischer Kenngrößen abzielen, vielleicht etwas im Schatten geblieben. Die Wasserwirtschaft widmet sich der Ordnung der Ansprüche zur Nutzung des Wassers, Schutz des Menschen vor dem Wasser und Schutz des Wassers vor den Menschen. Fast immer handelt es sich dabei um flächige Aussagen, also sind Punktdaten auf die Fläche zu extrapolieren - die zentrale Stärke von Fernerkundungsdaten. Österreich besitzt ein ausgezeichnetes hydrografisches Messnetz, vermutlich eines der besten der Welt. Dennoch lassen sich mit konventionellen Punktdaten nicht so weitreichende Aussagen treffen wie mit den flächigen Informationen, die die Fernerkundung liefert. Überflutungsflächen, Vegetation, Gletscherflächen, Bodenfeuchte, Schneedeckenkenngrößen, Niederschlag, Erosionsgebiete und morphologische Veränderung von Gewässern sind nur einige der zahlreichen Informationen, die uns Fernerkundungsmethoden liefern können, sei es terrestrisch, von Befliegungen oder von Satelliten. In der Satellitentechnologie hat sich in der Tat eine ähnliche Revolution wie beim Laserscanning abgespielt. Zahllose neue Satellitenplattformen sind in Umlauf und liefern genauere Daten als je zuvor. In vielen Forschungsaktivitäten in Österreich werden die neuesten Satellitendaten in aktuelle hydrologisch-wasserwirtschaftliche Methoden integriert, wie etwa im neuen, vom FWF geförderten Forschungsprogramm „Wasserwirtschaftliche Systeme", in dessen Rahmen in den nächsten 12 Jahren 70 DissertantInnen ausgebildet werden sollen (www.waterresources.at). Aber auch beim Operationalisieren von Satellitenprodukten - man denke nur daran, wie oft heute Google Earth benützt wird - hat sich sehr viel getan. EUMETSAT (The European Organisation for the Exploitation of Meteorological Satellites) hat etwa ein operationelles Datenservice gestartet, im Rahmen dessen Bodenfeuchtedaten in Echtzeit erhältlich sind. Ähnliche Initiativen existieren für Schneedaten und viele andere Satellitenprodukte, die für die Hydrologie und Wasserwirtschaft höchst relevant sind.

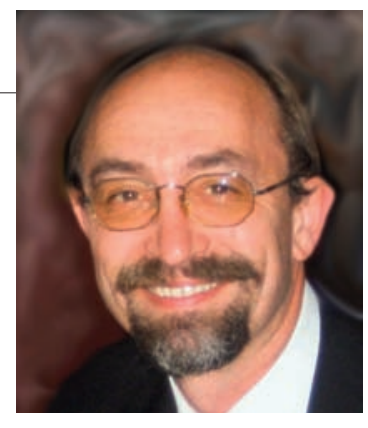

Univ.-Prof.

DI Dr. Günter Blöschl

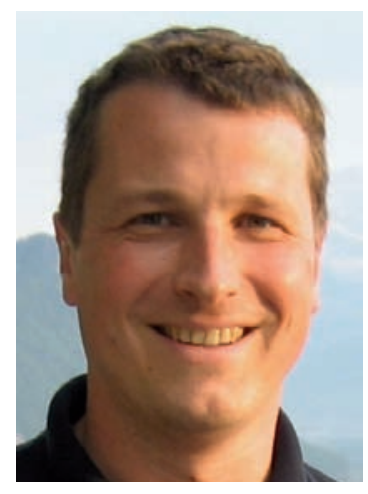

Univ.-Prof.

DI Dr. Wolfgang Wagner 


\section{editorial}

Bei all dieser Dynamik und Fülle neuer Information ist es wichtig, den Überblick zu behalten und die aktuellen Entwicklungen im Grundsatz nachvollziehen zu können. Das vorliegende Heft 07-08/2009 der Österreichischen Wasser- und Abfallwirtschaft soll einen Beitrag dazu leisten. Keinesfalls kann die Darstellung vollständig sein. Es wurde versucht, entsprechend der Bedeutung der einzelnen Methoden und Aufgaben, eine repräsentative Auswahl zu treffen. Vier Beiträge befassen sich mit Laserscanning bzw. mit der hydrodynamischen Modellierung (Mandlburger et al., Gabriel, Rauchlatner und Höppl, Habereder und Blaschke), wobei der letzte Beitrag zusätzlich auf die gekoppelte hydrodynamische Modellierung eingeht, zweifelsfrei ein Feld, das rasant an Bedeutung in Forschung und Praxis gewinnen wird. Zwei Beiträge (Hasenauer et al., Parajka et al.) beschäftigen sich mit der direkten Bestimmung hydrologischer Kenngrößen, nämlich Bodenfeuchte und Schneekenngrößen. Hier wird die Stärke der Fernerkundung, flächenhafte Daten zu liefern, besonders sichtbar. Mit dieser Auswahl würden sich die Unterzeichneten freuen, einen Beitrag zu den Bemühungen um gute Lösungsansätze für die Praxis leisten zu können.

\section{Univ.-Prof. DI Dr. Günter Blösch}

Technische Universität Wien

Institut für Wasserbau und Ingenieurhydrologie

Karlsplatz 13

1040 Wien

E-Mail: bloesch|@hydro.tuwien.ac.at

\section{Univ.-Prof. DI Dr. Wolfgang Wagner}

Technische Universität Wien

Institut für Photogrammetrie und Fernerkundung

Gußhausstraße 25-29

1040 Wien

E-Mail: ww@ipf.tuwien.ac.at 